\title{
A Heuristic Algorithm to Find Power Efficient Pre Configured Cycles (PEP-cycles) and Resolve NP Hard Issues
}

\author{
${ }^{1}$ Prerana, ${ }^{2}$ Rachna Asthana, ${ }^{3} \mathrm{M}$ Shukla \\ ${ }^{1-3}$ Department of Electronics Engineering, Harcourt Butler Technical University, Kanpur \\ Email:prerana.6289@gmail.com, rachnaasthana@rediffmail.com,manojkrshukla@gmail.com
}

Received: 03 ${ }^{\text {rd }}$ May 2019, Accepted: 13 $^{\text {th }}$ May 2019, Published: 30 $^{\text {th }}$ June 2019

\begin{abstract}
Now a days ICT has powerful impact on economy and environmental conditions of the country. The principle of green networking gives idea to design a energy aware network. These networks focus on the energy reduction and consumption of unnecessary energy to save the environment. It is predicted that the global IP traffic will be approx 63.9 Exabyte's per month in coming years .These increased bandwidth requires more bandwidth and it consumes large amount of energy and causes global warming too.

To reduce this global warming effect various power saving schemes are opted by the service providers that contributes to save the environment. In this scenario we develop a heuristic to choose the power efficient pre configured cycles (PEP- cycles) from the group of all cycles to minimize the effect of NP hard problem. The developed algorithm is used to achieve fast speed protection of optical network. The main aim of the algorithm is to reduce the complexity of the optimization and to resolve the complexity problem.
\end{abstract}

\section{Keywords}

Green Networking, Power Efficient P-Cycles (PEP-Cycles), NP Hard

\section{Introduction}

The population of world is increasing day by day and as a result the demand for energy requirement also increases. Large requirement of bandwidth and too many broad band applications along with high speed is the basic need of today's optical network scenario. The study shows that the network traffic has been growing by the annual rate of 45 percent since 2004 [1]. Such increase in traffic also concentrates in expanding the network capacity.

With the fast developing scenario of power consumption in Information and Communication Technology (ICT), power-efficient solutions in telecom networks have become necessary. Optical networks, as backbone of telecom networks, are also major contributor in the overall power consumption. Massive amount of data is transmitted through the network and the small failure in network can incur huge losses of data. The deployments of large number of redundant resources are required to achieve the goal of high reliable network architecture for the backup capacity for protection.

In recent years the effect of Green House Gasses (GHG) are the cause of green house effect. In atmosphere the increase in GHG increases the effect of global warming and climate change etc. As the report published by the European Union [2], It is needed to control the emission by $15 \%-30 \%$ before year 2020 in sake to control the global temperature increase below $2{ }^{\circ} \mathrm{C}$. Fig 1 shows the GHG emission report of year 2016 . The report shows that there was 6,511 Million Metric Tons of $\mathrm{CO}_{2}$ equivalent harmful gasses are released.

\section{U.S. Greenhouse Gas Emissions in 2016}

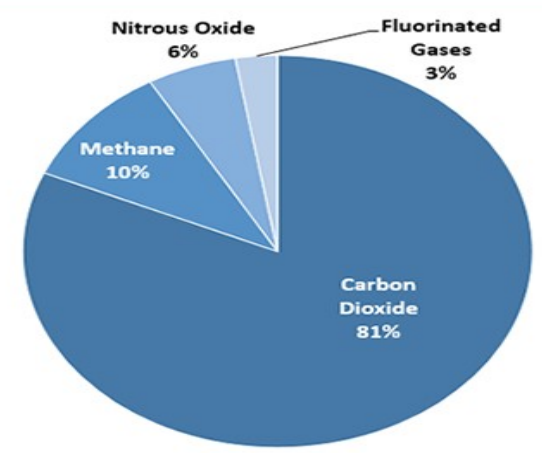

Figure 1: Emission Report of Gasses in 2016 
To reduce the energy consumption it is necessary to take some important steps that improves and minimizes the GHG emission and reduces the global warming for environmental point of view. Green networking is becoming popular day by day to save our environment and to reduce energy consumption.

ICT sector is $2 \%$ contributor in annual global greenhouse gases emissions [3]- [5]. According to 2007 annual report the internet alone shares $5.5 \%$ of total energy consumption. According to the data of 2009 , ICT consumes $8 \%$ of world's total electricity [6] [7]. The amount of power consumed by the centre networks is vast . Optical networks are the very heart of core network. The enormous bandwidth for the present and future applications of internet is provided by WDM networks. However in last few years the network designers and operators didn't paid much attention to power efficiency related issues. Therefore in current days many recent researches focus on powerefficient core network. For a better optical networks it is mandatory to combine up the two domains together that are survivability and Power -efficiency. These two domains are interlinked as the survivability of the network is targeted the requirement of redundant resources are increased and this undergoes a more consumption of power.

There is an urgent need to consider minimizing power consumption of optical networks and assuring survivability of network at the same time. Pre-Configured Cycles (p-cycles) are one of the most promising technique for shared protection. Unlike dedicated protection techniques, p-Cycles have combined advantage of fast restoration speed and efficiency.[8] It can be easily said that $p$ cycle protection technique is an hybrid mechanism of ring and mesh protection. P- cycle offers the protection to on cycle link as well as straddling links too. Straddling links are that links which does not belong to the protection cycle but their end nodes are on that cycle[9]. But for the large scale network and complicated network structures the pre configured cycles have some complexity issues to resolve this problem we developed a heuristic which finds the power efficient cycles that are more efficient to protect the network from the group of cycle. This heuristic reduces the complexity and time taking problem of the network In this paper we develop a heuristic to resolve the problem of NP hard for larger networks. We develop a algorithm to find the power efficient pre configured cycle for the network that reduces the complexity of the network. It provides easy solutions in practical computational time and better results in shorter running times

\section{Overview of the Wavelength Division Multiplexing Technique (WDM)}

Wavelength division multiplexing is one of the attractive technique of multiplexing for high speed network. The system employs numerous wavelengths, that are encoded individually with information and single mode fibres are used to multiplex them. These wavelengths are transmitted over the distance, and then wavelength demultiplexers are used to separate channels whose information is detected and received as shown in fig 2 . The system requires the regenerator for each wavelength at repeaters site therefore it doesn't implicated properly at initial stage. Compared with increasing capacity via Time division multiplexing (TDM), the approach did not scale capacity as cost effectively as TDM.[10]-[12]

WDM is used for the future telecommunication network by the carriers and service providers where increased network capacity is required. It is a feasible solution which utilizes the huge bandwidth provided by optical networks. Now a day's bandwidth capacity rate is increasing day by day and approaching approximately from several tens of terabits per second. These large scale systems make them complex and expensive too. In recent years things have changed. And the technology is evolving rapidly. The region of 1300 or $1550 \mathrm{~nm}$ spectrum band are used in WDM that is two wavelength windows at which optical fibers have very low signal loss.

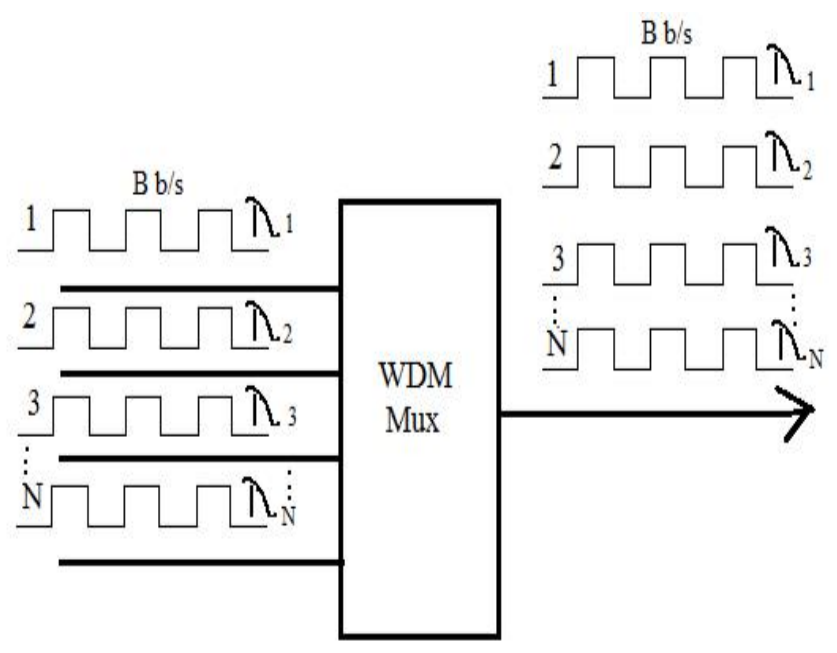

Figure 2: Wavelength Division Multiplexing (WDM) 


\section{Power Efficient P- cycle Heuristic}

In this section we heuristics to solve the NP hard problem. This heuristic describes and proves that it gives better performance results and the solving complexity of p-cycle is reduced. Generally we consider Hamiltonian cycle for the protection and it can be explained as the Hamiltonian path is such path that visits each and every vertex of the graph definitely for once. The starting and ending edge between the Hamiltonian path is called as Hamiltonian cycle. The Iris mathematician Sir William Rowan Hamilton found the concept of Hamiltonian cycle and path in a dodecahedron structure of twenty vertices and thirty edges [13].

Finding of Hamiltonian path and cycle is a general problem of researchers is known as NP-complete problem. We here briefly describe our proposed heuristic algorithm based on power efficiency principle. We used a bidirectional graph and the p-cycles of the graph covers all the spans and nodes at once. The concept of power efficient $\mathrm{p}$ cycle (PEP-cycle) is designed to get more optimal value with less complexity.

The heuristic method to find PEP-cycles has the following steps:

[1] Find all the p-cycles of the network by using Iterative Loop Counting Algorithm (ILCA). These p-cycles are the closed loop structures used to provide protection to a network.

[2] Randomly select the traffic i.e source and destination point in between the data has to be transferred.

[3] Search the route between the source and destination by using different path finding algorithm.

[4] Group all the cycles that goes through the span of the particular demand. The span of the traffic should be present in the network as the on cycle span or the straddling span.

[5] Now calculate the power efficiency of each individual cycle for a particular demand.

\section{Power efficiency $(\mathrm{PE})=\left(0.5 \sum\right.$ Number of spans covered by $p-$ cycle but not on the route of demand $)+$}

\section{$(1 *$ Enumber of on cycle span which are also the part of the traffic route $)+$} $\left(0.5^{*} \sum\right.$ Number of straddling span which is the part of the traffic route) / Total on cycle span

[6] Go to step [2] and repeat all the steps for each demand.

[7] Make the group of highest power efficient cycle and lowest power efficient cycle from all the data of power calculation of cycles of different demands.

\section{Result and Discussion}

We choose 8 node 11 span bi directional network as a test network as shown in Fig 3. We assume that weights of all spans are equal. By using ILCA, all the p-cycles for this $8 \mathrm{n} 11 \mathrm{~s}$ network are found. There are total $14 \mathrm{p}$ cycles. All the p-cycles are given in the below table.
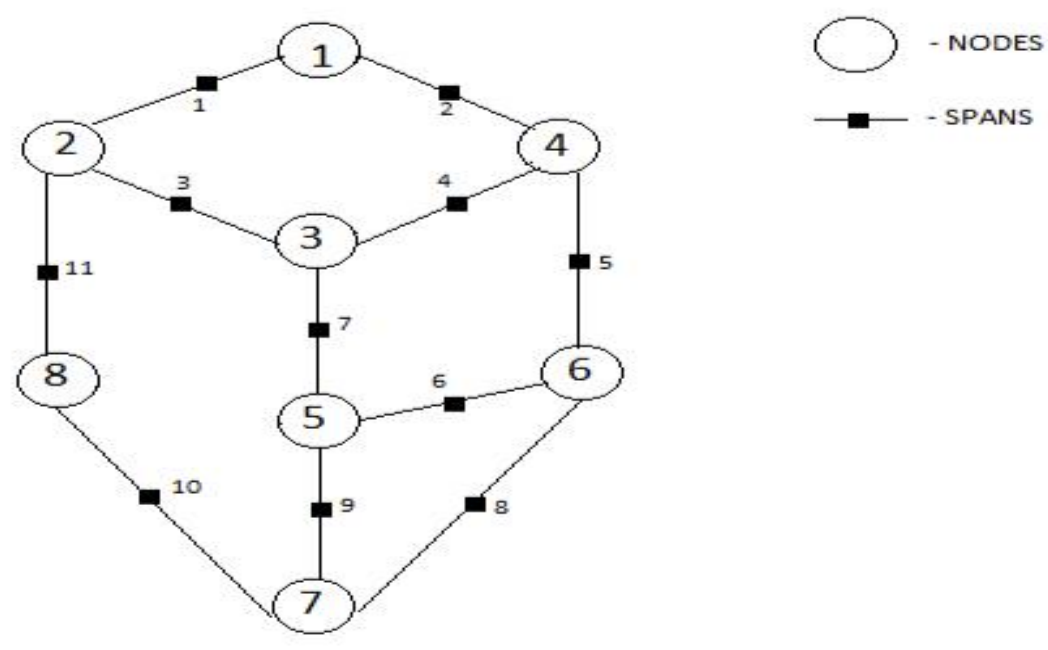

Figure 3: 8 Nodes 11 Spans Test Network 


\begin{tabular}{|c|c|c|c|}
\hline P-Cycle Number & $\begin{array}{c}\text { Path Which P-Cycle follows } \\
\text { (through nodes) }\end{array}$ & P-Cycle Number & $\begin{array}{c}\text { Path Which P-Cycle follows } \\
\text { (through nodes) }\end{array}$ \\
\hline P1 & $1-2-3-4-1$ & P8 & $2-3-4-6-5-7-8-2$ \\
\hline P2 & $1-2-3-5-6-4-1$ & P9 & $2-3-4-6-7-8-2$ \\
\hline P3 & $1-2-3-5-7-6-4-1$ & P10 & $2-3-5-6-7-8-2$ \\
\hline P4 & $1-2-8-7-5-3-4-1$ & P11 & $2-3-5-7-8-2$ \\
\hline P5 & $1-2-8-7-5-6-4-1$ & P12 & $3-4-6-5-3$ \\
\hline P6 & $1-2-8-7-6-4-1$ & P13 & $3-4-6-7-5-3$ \\
\hline P7 & $1-2-8-7-6-5-3-4-1$ & P14 & $5-6-7-5$ \\
\hline
\end{tabular}

Table 1: Path Followed by $p$-cycles of 8n11s Network (through nodes)

The above table 1 shows the path which is followed by each p-cycle. Now the calculation of power efficient cycle for the group of traffic demand is done and the maximum efficient and minimum efficient cycles are grouped individually. The given table 2 shows the p-cycle representation according their power efficiency.

\begin{tabular}{|l|l|l|}
\hline S. No & Categorization based on Power Efficiency & P-cycles \\
\hline 1. & Maximum power efficient cycle & P2,P3,P7,P8,P10,P12,P13 \\
\hline 2. & Minimum power efficient cycle & P2,P3,P5,P6,P8,P9,P10,P11,P12 \\
\hline
\end{tabular}

Table 2: Categorization of p-cycle Based on their Power Efficiency

The above table 2 shows that each demand follows the different group of p-cycles that traverses through the path of the demand. It can be possible that the cycle which has highest power efficiency in one demand may have lowest power in other demand as it totally depends on the path and the route which is followed.

\section{Conclusion}

It is very important to reduce the complexity and NP hard problem of large size networks because more energy and power is required to resolve the issues of complicated networks. Therefore, we use this heuristic to reduce the complexity of the network with more number of p-cycles. And from the group of $14 \mathrm{p}$-cycle the most power efficient 6 cycle are separated that gives better results with less complexity and shorter time.

\section{Acknowledgement}

The authors gratefully acknowledge financial support from the TEQUIP III World bank scheme. In addition, we wish to thank all the people related to the work who made valuable suggestions or who has otherwise contributed to the preparation of the manuscript.

\section{References}

[1] E. Le Rouzic, "Network Evolution and the Impact in Core Networks", Proc. ECOC, Turin, Italy, Sept. 2010.

[2] D. Pamlin and K. Szomol'anyi, "Saving the Climate @ the Speed of Light-First Roadmap for Reduced CO2 Emissions in the EU and Beyond." WorldWildlife Fund and European Telecommunications Network Operators' Association, Apr. 2007.

[3] Yabin Ye, Jorge Lo ’pez et al., “Energy-Efficient Resilient Optical Networks: Challenges and Trade-offs”, IEEE communication magazine, Feb 2015, pp. 144-150.

[4] Sofie Lambert, Ward Van Heddeghem, Willem Vereecken, Bart Lannoo, Didier Colle, and Mario Pickavet,

"Worldwide Electricity Consumption of Communication Networks", Optical Society of America publishing (OSA), Optics Express(2012), Vol.20, Issue26, pp- B513-B524.

[5] Bernardetta Addis, Antonio Capone, Giuliana Carello, Luca G. Gianoli, Brunilde Sanso, "Energy aware management of resilient networks with shared protection", Sustainable Internet And ICT For Sustainability (SustainIT) 2012,4-5 October 2012, IEEE, pp-1-9

[6] C. Cavdar, F. Buzluca, and L. Wosinska, "Energy-efficient design of survivable WDM networks with sharedbackup," Proc. Globecom (IEEE 2010).

[7]Rodney S. Tucker, " Green Optical Communications- Part II: Energy Limitations in Networks", IEEE Journal of Selected Topics in Quantum Electronics, vol. 17, No.2, March/April 2011, pp-261-274. 
[8] C. Liu and L. Ruan, "p-cycle design in survivable WDM networks with shared risk link groups (SRLGs)," Photonic Network Commun., vol. 11, no. 3, pp. 301-311, May 2006.

[9]M. Kiaei, C. Assi, and B. Jaumard, "A survey on the pcycle protection method," Communications Surveys \& Tutorials, IEEE, vol. 11, no. 3, pp. 53-70, 2009.

[10] Ramaswami, Sivarajan, Sasaki, "Optical Networks- A practical perspective”, Morgan Kaufmann (Elsevier) Publishers, 3rd Edition.

[11]. Gerd Keiser, “Optical Fiber Communication”, McGraw Hill Inc., 2nd Edition,1991.

[12] John M. Senior, “ Optical Fiber Communication- Principles and Practices”, Prentice Hall (India), 2nd Edition.

[13] A. S. Herschel. Sir Wm. Hamilton’s Icosian Game. Quart. J. Pure Applied Math, 5:305, 1862. 